\title{
Longterm Antiepileptic Therapy and Bone Health: Implications for Patients with Brain Tumors
}

\author{
Admir Mehičević ${ }^{1}$, Nevena Mahmutbegović 1 , Ibrahim Omerhodžić ${ }^{2}$, Enra Mehmedika Suljić ${ }^{1}$ \\ ${ }^{1}$ Neurology Clinic, Clinical Center, University of Sarajevo, Sarajevo, Bosnia and Herzegovina, ${ }^{2}$ Neurosurgery Clinic, Clinical \\ Center, University of Sarajevo, Sarajevo, Bosnia and Herzegovina \\ Correspondence: nevenaradulovic@hotmail.com; Tel.: + 38763284 178; Fax.: + 38733297821
}

Received: 26 April 2020; Accepted: 12 October 2020

\begin{abstract}
Objective. The objective of our study was to investigate the effects of carbamazepine (CBZ) and lamotrigine (LTG) treatment on bone metabolism in epileptic patients. Patients and Methods. A cross-sectional study was performed on normal controls $(\mathrm{N}=30)$ and 100 patients with symptomatic epilepsy caused by a primary brain tumor, divided into two groups according to the treatment: LTG monotherapy group $(\mathrm{N}=50)$ and $\mathrm{CBZ}$ monotherapy group $(\mathrm{N}=50)$. For each participant serum levels of 25-OHD and osteocalcin (OCLN) were measured, and bone mineral density (BMD) was evaluated by the dual-energy X-ray absorptiometry method. Results. There was no statistically significant difference in the average values of vitamin $\mathrm{D}$ in serum between the CBZ and LTG groups (Vitamin D CBZ 17.03 \pm 12.86 vs. Vitamin D LTG 17.97 $\pm 9.15 ; \mathrm{F}=0.171, \mathrm{P}=0.680$ ). There was no statistically significant difference in the average values of OCLN between the CBZ and LTG groups (OCLN CBZ 26.06 \pm 10.87 vs. OCLN LTG 27.87 $\pm 28.45 ; \mathrm{F}=0.171, \mathrm{P}=0.674)$. The $\mathrm{BMD}$ value was lower in both groups using antiepileptic agents compared to the controls, but when comparing the CBZ group to the LTG group, a statistically significant difference was only observed for the Z score (T-score CBZ: $0.08 \pm 1.38$ vs. T-score LTG: $0.37 \pm 1.02 ; \mathrm{F}=1.495, \mathrm{P}=0.224 ; \mathrm{Z}$ score CBZ: $-0.05 \pm 1.17$ vs. $\mathrm{Z}$. score CBZ: $0.38 \pm 0.96 ; \mathrm{F}=4.069, \mathrm{P}=0.046$ ) (Table 3). Conclusion. The choice of antiepileptic agents for treating seizures in patients with brain tumors should be carefully evaluated in relation to their impact on bone health. These patients could benefit from supplementation and regular measurement of biochemical markers of bone turnover and BMD.
\end{abstract}

Key Words: Osteoporosis • Anticonvulsants • Brain Neoplasms.

\section{Introduction}

Seizures are a common complication of both primary and metastatic brain tumors as they may appear as the initial manifestation or during the course of the disease (1). Such seizures are focal in origin and may either remain focal or secondarily generalize. As seizures represent an important source of morbidity and mortality in patients with primary and secondary brain tumors, they require aggressive treatment (2). Although carbamazepine (CBZ) has been considered as a first line agent for treating focal seizures with or without secondary generalization, antiseizure drugs with no or minimal hepatic enzyme-inducing properties, such as lamotrigine (LTG), are generally preferred, as these agents are considered to have a more favorable safety profile compared with older agents (3).

Long-term antiepileptic therapy has been strongly associated with negative effects on bone health. Multiple studies have reported the osteopenic effect of CYP450 inducing antiepileptic drugs (AEDs), reflected in hypocalcemia, hypophosphatemia, increased vitamin D catabolism, hyperparathyroidism, elevated markers of bone resorption and accelerated bone mass loss (4-6). Novel studies have reported that AEDs may cause bone loss in the absence of vitamin D deficiency (7). However, data on newer AEDs are insufficient, and although their use has become increasingly prevalent, their effects on bone metabolism remain poorly understood. 
It has been proposed that patients with brain tumors, particularly those with a long-term life expectancy, may benefit from regular checks of bone turnover markers and BMD testing (8). Therefore, the choice of antiepileptic agents in this specific group of patients should include a critical assessment of their impact on bone health. The objective of our study was to investigate the effects of carbamazepine (CBZ) and lamotrigine (LTG) treatment on bone metabolism in epileptic patients.

\section{Patients and Methods}

A cross-sectional study was performed between 2016 and 2018, at the Neurology Clinic in Sarajevo. We prospectively recruited 100 patients with symptomatic epilepsy caused by a primary brain tumor. The subjects in the case group were further stratified according to treatment into two different patient groups: epileptic patients undergoing treatment with LTG monotherapy $(\mathrm{N}=50)$, and $\mathrm{CBZ}$ monotherapy $(\mathrm{N}=50)$ for a period of at least twelve months. Patients who had any condition known to affect bone metabolism (e.g., renal disease, recent fracture, hyperparathyroidism, Paget disease, osteoporosis) or who were taking any drug known to cause or treat osteoporosis, were excluded. We also excluded patients with diagnosed metastatic disease and patients who had received AEDs before the presumptive diagnosis of brain tumor. The results were compared with age-matched healthy controls with no evidence of metabolic bone disease $(\mathrm{N}=30)$.

All participants were asked to complete a questionnaire including medical history, information on fractures, falls and injuries, and vitamin $\mathrm{D}$ or calcium supplements. Bone mineral density (BMD) was evaluated by a dual-energy X-ray absorptiometry method called DXA technology. DXA was performed using a Hologic QDR-4000A densitometer (Hologic, Bedford, MA, U.S.A.). DXA measured bone mineral content (BMC in grams) and bone area (BA, in square centimeters), then calculated BMD "area" in $\mathrm{g} / \mathrm{cm} 2$ by dividing $\mathrm{BMC}$ by $\mathrm{BA}$. The T-score, the value used to diagnosis osteoporosis, is defined as the difference be- tween the patient's $\mathrm{BMD}$, and the mean reference value of the normal young population, divided by the standard deviation of the normal young population. The Z-score, used to compare the patients' BMD with a population of peers, is calculated by subtracting the mean BMD of an age, ethnicity and sex-matched reference population from the patients' BMD, and dividing it by the SD of the reference population.

For each subject, the level of vitamin D and osteocalcin (OCLN) in the serum was determined in laboratory findings. Serum 1, 25-dihydroxyvitamin D (3) (normal range, 20-74 pg/ml) was measured by radioimmunoassay. Serum osteocalcin level was determined by Elisa. Due to laboratory errors, not every test was obtained for every patient. The precise $\mathrm{n}$ for each test in each patient group is noted in the tables.

\section{Ethics Statement}

The study was conducted according to the standards of the Declaration of Helsinki (1975, revised 2000), and the protocol was approved by the local Bioethical Committee (decision reference numbers 0207-28784).

\section{Statistical Analysis}

Statistical analyses were performed using SPSS for Windows, version 16 (SPSS Inc., Chicago, IL). Continuous data were presented as mean \pm standard deviation (SD). Student's t-test and ANOVA test were used for continuous variables as baseline comparisons between the cases and the controls. Odds ratio (OR) and 95\% confidence interval (CI) were calculated. A P value of $\leq 0.05$ was considered significant.

\section{Results}

The study involved 50 subjects on CBZ monotherapy with mean age $36.74 \pm 10.26$ years, and 50 subjects on LTG monotherapy with mean age $31.82 \pm 8.84$ years, compared to the age matched controls. The characteristics of the study partici- 
pants are shown in Table 1. We observed a statistically significant difference in the gender structure between the LTG group and the controls $(\chi 2=4.301$; $\mathrm{P}=0.033)$. The average duration of epilepsy in the CBZ group was $11.18 \pm 6.87$ years and in the LTG group $7.28 \pm 4.47$ years. The average duration of CBZ therapy was $6.18+3.36$ years. The average duration of LTG therapy was $4.72 \pm 2.52$ years. A statistically significant difference in the duration of epilepsy was found ( $\mathrm{F}=12.151 ; \mathrm{P}=0.001$.), as well as in the duration of therapy $(\mathrm{F}=6.012 ; \mathrm{P}=0.016)$ (Table 1).

Although the average value of vitamin $\mathrm{D}$ in serum was significantly lower in both groups using antiepileptic agents compared to the controls, there was no statistically significant difference between the CBZ and LTG groups (Vitamin D CBZ $17.03 \pm 12.86$ vs. Vitamin D LTG 17.97 \pm 9.15 ;
$\mathrm{F}=0.171, \mathrm{P}=0.680$ ) (Table 2). Although the average value of OCLN in serum was higher in both groups using antiepileptic agents compared to the controls, there was no statistically significant difference between the CBZ and LTG groups (OCLN CBZ 26.06 \pm 10.87 vs. OCLN LTG 27.87 \pm 28.45 ; $\mathrm{F}=0.171, \mathrm{P}=0.674)$ (Table 2).

The BMD values were lower in both groups using antiepileptic agents compared to the controls, but when comparing the CBZ group to the LTG group, a statistically significant difference was only observed for the $\mathrm{Z}$ score (T-score CBZ: $0.08 \pm 1.38$ vs. T-score LTG: $0.37 \pm 1.02 ; \mathrm{F}=1.495, \mathrm{P}=0.224 ; \mathrm{Z}$ score $\mathrm{CBZ}$ : $-0.05 \pm 1.17$ vs. $Z$. score LTG: $0.38 \pm 0.96$; $F=4.069$, $\mathrm{P}=0.046$ ) (Table 3 ). In regression analysis, the $\mathrm{Z}$ score was significantly associated with the duration of antiepileptic therapy $(\mathrm{F}=8.438, \mathrm{P}=0.005)$ (Table 4).

Table 1. Clinical Characteristics of Patients and Controls

\begin{tabular}{|c|c|c|c|c|c|c|c|c|}
\hline Variables & & $\mathrm{N}$ & $(\bar{x} \pm S D)$ & SEM & Minimum & Maximum & $\mathrm{F}$ & $\mathrm{P}$ \\
\hline \multirow[t]{3}{*}{ Age (yr) } & Controls & 30 & $40.37 \pm 8.30$ & 1.52 & 22.0 & 50.0 & $2.688^{*}$ & $0.105^{*}$ \\
\hline & $\mathrm{CBZ}$ & 50 & $36.74 \pm 10.26$ & 1.45 & 20 & 57.00 & $18.329^{\dagger}$ & $0.0001^{\dagger}$ \\
\hline & LTG & 50 & $31.82 \pm 8.84$ & 1.25 & 19.00 & 54.00 & $6.599^{\ddagger}$ & $0.012^{\ddagger}$ \\
\hline \multirow{3}{*}{$\begin{array}{l}\text { Gender (M), } \\
\mathrm{N}(\%)\end{array}$} & Controls & $16(53.3)$ & - & - & - & - & - & $0.177^{*}$ \\
\hline & $\mathrm{CBZ}$ & $20(40.0)$ & - & - & - & - & - & $0.033^{+}$ \\
\hline & LTG & $15(30.0)$ & - & - & - & - & - & $0.201^{\ddagger}$ \\
\hline \multirow{2}{*}{$\begin{array}{l}\text { Duration of } \\
\text { epilepsy (yr) }\end{array}$} & CBZ & 49 & $11.18 \pm 6.87$ & 0.98 & 2.00 & 35.00 & - & - \\
\hline & LTG & 50 & $7.28 \pm 4.47$ & 0.63 & 2.00 & 23.00 & $11.217^{\ddagger}$ & $0.001^{\ddagger}$ \\
\hline \multirow{2}{*}{$\begin{array}{l}\text { Duration of } \\
\text { antiepileptic } \\
\text { treatment } \\
\text { (yr) }\end{array}$} & $\mathrm{CBZ}$ & 49 & $6.18 \pm 3.36$ & 0.48 & 2.00 & 15.00 & - & - \\
\hline & LTG & 50 & $4.72 \pm 2.52$ & 0.36 & 2.00 & 12.00 & $6.012 \ddagger$ & $0.016^{\ddagger}$ \\
\hline
\end{tabular}

$\mathrm{CBZ}=$ Carbamazepine; $\mathrm{LTG}=$ Lamotrigine; ${ }^{*}$ For comparison of controls and CBZ group; ${ }^{+}$For comparison of controls and LTG group; ${ }^{*}$ For comparison of CBZ and LTG groups.

Table 2. Average Values of 25-Hydroxyvitamin Vitamin D and OCLN in Serum in Patients and the Control Group

\begin{tabular}{lllllllll}
\hline Biochemical markers & $\mathrm{N}$ & $(\overline{\mathrm{x}} \pm \mathrm{SD})$ & SEM & Minimum & Maximum & $\mathrm{F}$ & $\mathrm{P}$ \\
\hline \multirow{2}{*}{ Vitamin D $(\mathrm{ng} / \mathrm{ml})$} & Controls & 30 & $32.03 \pm 6.99$ & 1.28 & 21.30 & 50.30 & $14.440^{*}$ & $0.0001^{*}$ \\
\cline { 2 - 9 } & CBZ & 49 & $17.03 \pm 12.86$ & 1.86 & 3.00 & 72.90 & $12.146^{+}$ & $0.0001^{\dagger}$ \\
\cline { 2 - 9 } & LTG & 50 & $17.97 \pm 9.15$ & 1.31 & 4.10 & 39.10 & $0.171^{\ddagger}$ & $0.680^{\ddagger}$ \\
\hline \multirow{2}{*}{ OCLN $(\mathrm{ng} / \mathrm{ml})$} & Controls & 30 & $19.64 \pm 6.54$ & 1.19 & 11.10 & 36.40 & $8.671^{*}$ & $0.004^{*}$ \\
\cline { 2 - 9 } & CBZ & 50 & $26.06 \pm 10.78$ & 1.52 & 11.90 & 77.10 & $2.422^{+}$ & $0.124^{\dagger}$ \\
\cline { 2 - 9 } & LTG & 50 & $27.87 \pm 28.45$ & 4.02 & 16.00 & 221.00 & $0.178^{\ddagger}$ & $0.674^{\ddagger}$ \\
\hline
\end{tabular}

OCLN=osteocalcin; CBZ=carbamazepine; LTG=lamotrigine; ${ }^{*}$ For comparison of controls and CBZ group; ${ }^{\dagger}$ For comparison of controls and LTG group; ${ }^{\star}$ For comparison of CBZ and LTG groups. 
Acta Medica Academica 2020;49(Suppl 1):S23-29

Table 3. BMD Values in Patients and the Control Group

\begin{tabular}{|c|c|c|c|c|c|c|c|c|}
\hline \multicolumn{2}{|c|}{ BMD values } & \multirow{2}{*}{$\begin{array}{l}\mathrm{N} \\
30\end{array}$} & \multirow{2}{*}{$\begin{array}{l}(\bar{x} \pm S D) \\
0.73 \pm 1.13\end{array}$} & \multirow{2}{*}{$\begin{array}{l}\text { SEM } \\
0.21\end{array}$} & \multirow{2}{*}{$\begin{array}{l}\text { Minimum } \\
-2.70\end{array}$} & \multirow{2}{*}{$\begin{array}{l}\text { Maximum } \\
2.10\end{array}$} & \multirow{2}{*}{$\begin{array}{l}\mathrm{F} \\
4.853^{*}\end{array}$} & \multirow{2}{*}{$\begin{array}{l}P \\
0.031^{*}\end{array}$} \\
\hline Tscore & Controls & & & & & & & \\
\hline & CBZ & 49 & $0.08 \pm 1.38$ & 0.20 & -3.40 & 3.90 & $2.158^{+}$ & $0.146^{+}$ \\
\hline & LTG & 50 & $0.37 \pm 1.03$ & 0.14 & -2.70 & 2.40 & $1.495^{\ddagger}$ & $0.224^{\ddagger}$ \\
\hline \multirow[t]{3}{*}{ Z score } & Controls & 30 & $0.55 \pm 0.79$ & 0.14 & -1.00 & 1.80 & $6.750^{*}$ & $0.015^{*}$ \\
\hline & CBZ & 49 & $-0.05 \pm 1.17$ & 0.17 & -3.10 & 1.50 & $0.663^{+}$ & $0.418^{+}$ \\
\hline & LTG & 50 & $0.38 \pm 0.96$ & 0.14 & -3.30 & 2.10 & $4.069^{\ddagger}$ & $0.046^{\ddagger}$ \\
\hline
\end{tabular}

$\mathrm{BMD}=$ bone mineral density; $\mathrm{CBZ}=$ carbamazepine; $\mathrm{LTG}=$ lamotrigine; "For comparison of controls and CBZ group; ${ }^{\dagger}$ For comparison of controls and $\mathrm{LTG}$ group; ${ }^{\ddagger}$ For comparison of CBZ and LTG groups.

Table 4. Logistic Regression Analysis and Risk Factors Independently Associated with Z Score

\begin{tabular}{llllll}
\hline Tests of between-subjects effects & & & & & \\
\hline Dependent variable: $Z$ score & & & & & \\
\hline Source & $\begin{array}{l}\text { Type III } \\
\text { Sum of Squares }\end{array}$ & df & $\begin{array}{l}\text { Mean } \\
\text { square }\end{array}$ & Sig. \\
\hline Gender & 0.282 & 1 & 0.282 & 0.314 & 0.576 \\
\hline Duration of epilepsy & 0.788 & 1 & 0.788 & 0.877 & 0.351 \\
\hline Duration of antiepileptic treatment & 7.578 & 1 & 7.578 & 8.438 & 0.005 \\
\hline
\end{tabular}

\section{Discussion}

Seizures are a frequent and overwhelming complication of brain tumors, with severe clinical and social effects, and a major negative impact on the quality of life. Additionally, the presence of epileptic seizures is considered to be associated with the long-term disability of patients with brain tumors (9). It has been reported that seizures are the initial manifestation of $20-45 \%$ of brain tumors, while another $20-45 \%$ of patients will present with them during the longer course of the disease (9).

The choice of antiepileptic agent in patients with brain tumors is mostly based upon its efficacy, tolerability and pharmacokinetic interactions (10). It has been reported that patients with brain tumors experience more side-effects of antiepileptic drugs compared to the overall population of epileptic patients, particularly bone-marrow suppression. Additionally, brain tumor-related seizures are often poorly controlled, and require the concomitant use of multiple AED, which leads to an even a higher risk of bone disease $(11,12)$.
Several mechanisms have been proposed to explain the negative effects of antiepileptic treatment on bone mineralization. Induction of the cytochrome P-450 system, resulting in alteration of vitamin $\mathrm{D}$ metabolism, is a widely accepted explanation $(4-6,13,14)$. The increased vitamin $\mathrm{D}$ catabolism leads to consequent hypocalcaemia and secondary hyperparathyroidism, increased bone resorption and accelerated bone mass loss. This mechanism is primary linked to the use of phenobarbital, phenytoin, and carbamazepine. Several studies report the osteopenic effects of carbamazepine on bone metabolism (15-18). On the other hand, several authors did not observe negative effects of carbamazepine therapy on vitamin D levels or biochemical markers of bone turnover $(19,20)$.

Moreover, several studies have also suggested that non-P-450 system mechanisms may play a role in bone loss (21). A large broad-spectrum of new generation AEDs, such as lamotrigine, topiramate, tiagabine, levetiracetam, oxcarbazepine, and zonisamide, have been considered to have fewer negative effects on bone health. Meyer et al. concluded that the molecule of lamotrigine shows 
good penetration into tumor tissues and therefore might be efficacious in brain tumor patients, and even, due to its excellent tolerability and auspicious safety profile, it might be considered as the first choice agent for treating seizures in these patients (22). Nevertheless, the unfavorable effects of LTG on bone, including bone loss, impaired growth in children, alterations in BMD, and elevated bone turnover markers, have been reported $(16,23,24)$.

In this study, we demonstrated a significant decrease in 25-OHD levels in patients treated with antiepileptic therapy compared to the control group. However there was no statistically significant difference between the CBZ and LTG groups (Vitamin D CBZ $17.03 \pm 12.86$ vs. Vitamin D LTG 17.97 $\pm 9.15 ; \mathrm{F}=0.171, \mathrm{P}=0.680$ ) (Table 2). Also, the average value of OCLN in serum was higher in both groups using AEDS compared to the controls, but there was no statistically significant difference between the CBZ and LTG groups (OCLN CBZ 26.06 \pm 10.87 vs. OCLN LTG $27.87 \pm 28.45$; $\mathrm{F}=0.171, \mathrm{P}=0.674$ ) (Table 2).

Malignancy itself, treatments and their consequences, malnourishment, and limited motion likely all affect BMD (25). Previous research has shown that brain tumor survivors are at higher risk of bone fractures and early osteoporosis (26). Several authors have explored bone diseases related to the use of AEDs, radiation therapy, anticoagulants, chemotherapy, and hemiplegia-associated osteopenia (8).

According to our results, BMD values were lower in both groups using antiepileptic agents compared to the controls, but when comparing the CBZ group to the LTG group, a statistically significant difference was only observed for the $\mathrm{Z}$ score ( $\mathrm{T}$ score CBZ: $0.08 \pm 1.38$ vs. T-score LTG: $0.37 \pm 1.02$; $\mathrm{F}=1.495, \mathrm{P}=0.224 ; \mathrm{Z}$ score $\mathrm{CBZ}:-0.05 \pm 1.17$ vs. $\mathrm{Z}$. score LTG: $0.38 \pm 0.96 ; \mathrm{F}=4.069, \mathrm{P}=0.046$ ) (Table 3). However, we also observed a statistically significant difference in the duration of epilepsy ( $\mathrm{F}=12.151 ; \mathrm{P}=0.001$ ), as well as in the duration of therapy $(\mathrm{F}=6.012 ; \mathrm{P}=0.016)$ (Table 1$)$. After adjustment, the $\mathrm{Z}$ score was significantly associated with the duration of antiepileptic therapy $(\mathrm{F}=8.438$, $\mathrm{P}=0.005$ ) (Table 4). The duration of antiepileptic treatment has been widely recognized as an independent predictor of lower BMD values (7).

A recent publication studied the use of AEDs and the risk of fracture, and concluded that longterm use of AEDs, particularly in women, was associated with a higher risk of bone fractures, but did not observe any differences between users of AEDs that do and do not induce the cytochrome P-450 system (21). In our study, we only observed a statistically significant difference in the gender structure between the LTG group and the controls $(\chi 2=4.301 ; \mathrm{P}=0.033)$ (Table 1$)$. In both groups using AEDs the female examinees dominated $(\chi 2=1.099 ; \mathrm{P}=0.201)$ (Table 1). The female ratio in the group with LTG was slightly higher compared to the CBZ group, which can be explained by the fact that LTG as a newer generation is preferred in women in the germinal period because of the low risk for developing congenital malformations. Both female gender and the postmenopausal period are significant risk factors for bone loss, however, young females have estrogen protection for bone metabolism (27). Pack et al. suggested that the bone loss observed in premenopausal women was associated with lower levels of estrogen (28). Unfortunately, in our study, we did not explore the levels of reproductive hormons in our patients, which may represent a major limitation.

It has been proposed that all patients with brain tumors who are initiating treatment should undergo BMD testing, particularly those with tumors compatible with a long-term life expectancy (8). Female gender may present a particularly vulnerable subgroup of these patients. Patients with verified osteopenia and osteoporosis should be treated, and it seems reasonable to avoid use of cytochrome P-450 AEDs in these patients for treating seizures (8). Further investigation of the effects of new generation AEDs on bone metabolism is necessary.

\section{Conclusion}

The choice of antiepileptic agents for treating seizures in patients with brain tumors should be carefully evaluated regarding their impact on bone 
health. These patients could benefit from supplementation and regular measurement of biochemical markers of bone turnover and BMD.

\section{What Is Already Known on this Topic:}

Long-term antiepileptic therapy, especially with enzyme-inducing agents, is associated with a high risk of bone disease. Therefore, regular osteoporosis investigation is recommended for these patients.

\section{What this Study Adds:}

In patients with brain tumors and symptomatic epileptic seizures, cautious use of AEDs should be kept in mind in order to avoid complications from bone loss. Preventive strategies, including regular supplementation and control of biochemical and radiological evidence of bone disease, should be implemented.

Authors' Contributions: Conception and design: AM and $\mathrm{NM}$; Acquisition, analysis and interpretation of data: AM, NM, IO and EMS; Drafting the article: AM and NM; Revising it critically for important intellectual content: IO and EMS; Approved final version of the manuscript: IO and EMS.

Conflict of Interest: The authors declare that they have no conflict of interest.

\section{References}

1. Avila EK, Graber J. Seizures and epilepsy in cancer patients. Curr Neurol Neurosci Rep. 2010;10(1):60-7.

2. Maschio M, Aguglia U, Avanzini G, Banfi P, Buttinelli C, Capovilla G, et al. Management of epilepsy in brain tumors. Neurol Sci. 2019;40(10):2217-34

3. Pilotto C, Liu JF, Walker DA, Whitehouse WP. Seizure characteristics and the use of anti-epileptic drugs in children and young people with brain tumours and epileptic seizures: Analysis of regional paediatric cancer service population. Seizure. 2018;58:17-21.

4. Miziak B, Błaszczyk B, Chroscinska-Krawczyk M, Danilkiewicz G, Jagiełło-Wójtowicz E, Czuczwar SJ. The problem of osteoporosis in epileptic patients taking antiepileptic drugs. Expert Opin Drug Saf. 2014;13(7):935-46.

5. Gallagher JC, Sai AJ. Vitamin D insufficiency, deficiency, and bone health. J Clin Endocrinol Metab. 2010;95(6):2630-3.

6. Wang Z, Schuetz EG, Xu Y, Thummel KE. Interplay between vitamin $\mathrm{D}$ and the drug metabolizing enzyme CYP3A4. J Steroid Biochem Mol Biol. 2013;136:54-8.

7. Arora E, Singh H, Gupta YK. Impact of antiepileptic drugs on bone health: Need for monitoring, treatment, and prevention strategies. J Family Med Prim Care. 2016;5(2):248-53.

8. Da Silva AN, Heras-Herzig A, Schiff D. Bone health in patients with brain tumors. Surg Neurol. 2007;68(5):525-33.
9. Maschio M. Brain tumor-related epilepsy. Curr Neuropharmacol. 2012;10(2):124-33.

10. Gefroh-Grimes HA, Gidal BE. Antiepileptic drugs in patients with malignant brain tumor: beyond seizures and pharmacokinetics. Acta Neurol Scand. 2016;133(1):4-16.

11. Kargiotis O, Markoula S, Kyritsis AP. Epilepsy in the cancer patient. Cancer Chemother Pharmacol. 2011;67(3):489501.

12. Laghari AA, Ahmed SI, Qadeer N, Shamim MS. Choice of therapeutic anti-seizure medication in patients with brain tumour. J Pak Med Assoc. 2019;69(3):442-4.

13. Maguire M, Marson AG, Ramaratnam S. Epilepsy (generalised). BMJ Clin Evid. 2010;2010:1201.

14. Fan HC, Lee HS, Chang KP, Lee YY, Lai HC, Hung PL, et al. The Impact of Anti-Epileptic Drugs on Growth and Bone Metabolism. Int J Mol Sci. 2016;17(8):1242.

15. Wang Z, Lin YS, Dickmann LJ, Poulton EJ, Eaton DL, Lampe JW, et al. Enhancement of hepatic 4-hydroxylation of 25-hydroxyvitamin D3 through CYP3A4 induction in vitro and in vivo: Implications for drug-induced osteomalacia. J Bone Miner Res. 2013;28(5):1101-16.

16. Zhang X, Zhong R, Chen Q, Li M, Lin W, Cui L. Effect of carbamazepine on the bone health of people with epilepsy: a systematic review and meta-analysis. J Int Med Res. 2020;48(3):300060520902608.

17. Rahimdel A, Dehghan A, Moghadam MA, Ardekani AM. Relationship between Bone Density and Biochemical Markers of Bone among Two Groups Taking Carbamazepine and Sodium Valproate for Epilepsy in Comparison with Healthy Individuals in Yazd. Electron Physician. 2016;8(11):3257-3265.

18. Verrotti A, Greco R, Latini G, Morgese G, Chiarelli F. Increased bone turnover in prepubertal, pubertal, and postpubertal patients receiving carbamazepine. Epilepsia. 2002;43(12):1488-92.

19. Turan MI, Cayir A, Ozden O, Tan H. An examination of the mutual effects of valproic acid, carbamazepine, and phenobarbital on 25-hydroxyvitamin D levels and thyroid function tests. Neuropediatrics. 2014;45(1):16-21.

20. Kir HM, Garip Ş, Şahin D, Öztaș B. Effects of carbamazepine on serum parathormone, 25-hydroxyvitamin D, bone specific alkaline phosphatase, C-telopeptide, and osteocalcin levels in healthy rats. Bosn J Basic Med Sci. 2012;12(4):240-4.

21. Souverein PC, Webb DJ, Weil JG, Van Staa TP, Egberts AC. Use of antiepileptic drugs and risk of fractures: casecontrol study among patients with epilepsy. Neurology. 2006;66(9):1318-24.

22. Meyer FP, Banditt P, Schubert A, Schöche J. Lamotrigine concentrations in human serum, brain tissue, and tumor tissue. Epilepsia.1999;40(1):68-73.

23. Lee HS, Wang SY, Salter DM, Wang CC, Chen SJ, Fan HC. The impact of the use of antiepileptic drugs on the growth of children. BMC Pediatr. 2013;13(1):211. 
24. Guo CY, Ronen GM, Atkinson SA. Long-term valproate and lamotrigine treatment may be a marker for reduced growth and bone mass in children with epilepsy. Epilepsia. 2001;42(9):1141-7.

25. Ferioli M, Zauli G, Martelli AM, Vitale M, McCubrey JA, Ultimo S, et al. Impact of physical exercise in cancer survivors during and after antineoplastic treatments. Oncotarget. 2018;9(17):14005-34.
26. Wilson CL, Ness KK. Bone mineral density deficits and fractures in survivors of childhood cancer. Curr Osteoporos Rep. 2013;11(4):329-37.

27. Sazgar M. Treatment of Women With Epilepsy. Continuum (Minneap Minn). 2019;25(2):408-30.

28. Pack AM, Morrell MJ, McMahon DJ, Shane E. Normal vitamin $\mathrm{D}$ and low free estradiol levels in women on enzyme-inducing antiepileptic drugs. Epilepsy Behav. 2011;21(4):453-8. 